\title{
Aufklärungsblatt der radiotherapeutischen Klinik der Universität Zürich
}

A llgemeine Wamzeichen:

Abmagerung.

Chronische Blutarmut. KaiTipf dGm Krebs! 3- Andauernde Miidigkeit.

4. Plotzliehes Auftreten von Knoten am Korper. Merke: Fieber und Schmerzen fehlen im Anfang!

Besondere Wamzeichen:

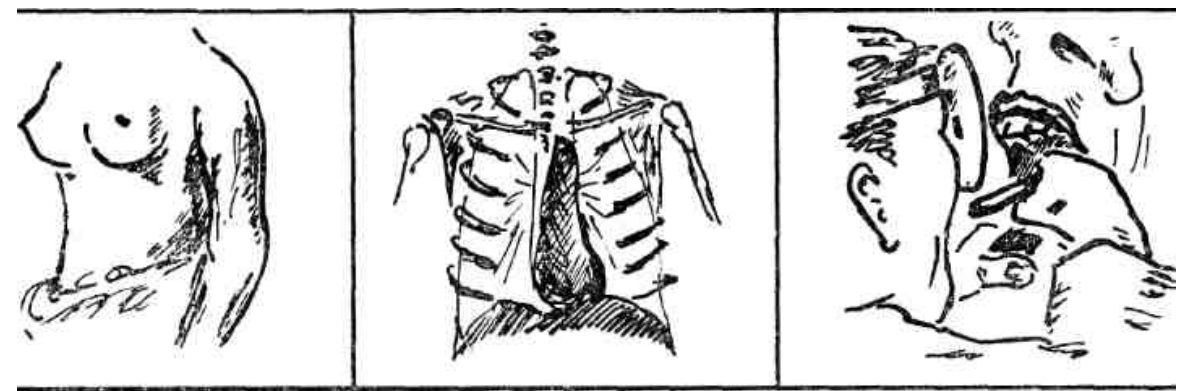

Schmerzlose Verhartung und Knoten Andauernder Husten, blutiger Andauernde Heiserkeit, Fremdkor

in der Brust. Blntende Brust. Auswurf. Atemnot. pergefuhl und nichtheilende Geschwiire in Mund und Hals.

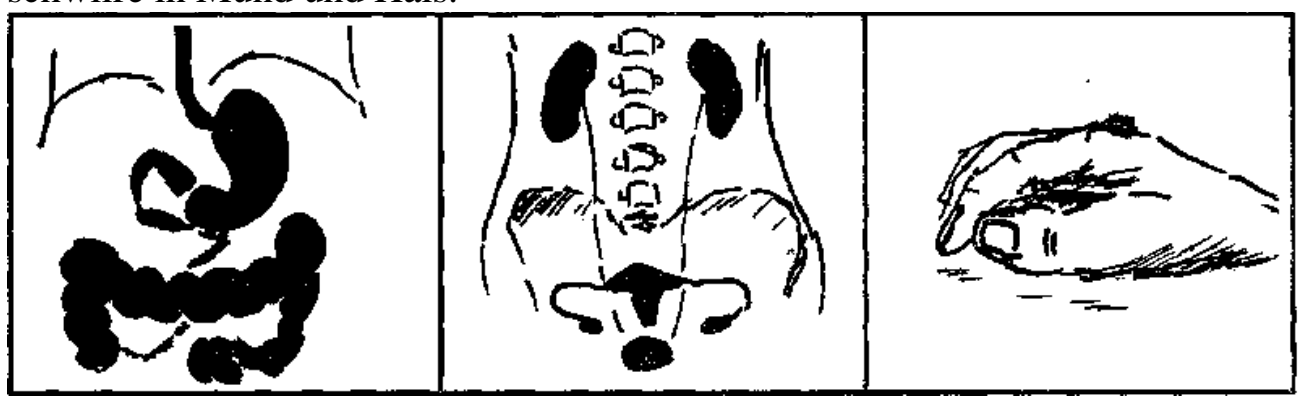

Andauernde Schluckbeschwerden, BlutimUrin, Menstruations- Nichtheilende Hautgesehwiire, plotz-

Appetitlosigkeit, Verdauungsstorun- storungen, Blutungen nach liehes Wachstum von Warze und

gen, Durehfalle, Verstopfung, Blut Abanderung. Muttermal.

im Stuhl.

Bei Auftreten soldier Wamzeichen ist drztliche und spezialarztliche Untersuchung unerlafilich

Krebs ist heilbar.

Vorbedingungen der Heilung ist Fruherfassung, Friiherkennung tmd Friihbeliandlung. Ohne arztliche Hilfe fiihrt der Krebs immer zum Tod. 
Einzige Mittel zur Krebslieilung sind Operation und Strahlenbehandlung (Rontgen- und Radiumstrahlen).

Auch bei fortgesehrittenem Krebs ist Linderung des Leidens moglich.

Jedem Krebskranken kann arztliche Hilfe geboten werden.

Der Krebskranke ist nicht ansteckend und bildet keine Gefahr fiir die Umgebung.

Prof. Dr. H. R. Schinz

Aufklarungsblatt der radiotherapeutischen Klinik der Universitat Zurich

Zehn Thesen zur Krebsvorbeugung

Allgemeine Korperhygiene und sorgf altige Mundpflege sowie gesunde Ernahrungsweise mit gemischter vitaminhaltiger Kost wirken vorbeugend. Einseitige Ernfihrung und Uberbelastung des Stoffwechsels sind zu vermeiden, ebenso zu groBer Alkoholkonsum. Ubertriebene Sonnenbestrahlung kann schadlich wirken.

Chroiiische Schadigungen wie Geschwiire und kleine, anfanglich harmlose Wucherungen sind amtlich zu behandeln.

Jugendliche sind vor dem Zigarettenrauchen und vor allem vor dem Inhalieren zu warnen. Die Verschmutzung der atmospharischen Luft mit Rauch, Motoren- und Fabrikabgasen ist zu bekampfen.

Die Bleichung, Farbung und Konservierung von Nahrungs- und GenuBmitteln darf nur mit Substanzen erfolgen, welche in langdauernden Tierversuchen auf cancerogene Wirkung gepriift und als unschadlich befunden worden sind. Dasselbe gilt fur Toiletten-artikel und Kosmetika. Alle Zusatze sollten deklariert werden.

Schadlingsbekampfungsmittel und Diingemittel sind vor Verwendung auf allfallige cancerogene Wirkung zu priifen. Solche Riickstande an Friichten und Gemiisen konnten gefahrlich sein. Chemische Krebse in Fabriken konnen durch Fabrikhygiene, insbesondere durch Ent-staubung und Entliiftung bekampft werden.

Strahlenkrebse durch Rontgenstrahlen und radioaktive Isotope miissen durch strengste Schutzvorschriften imd SchutzmaBnahmen vermieden werden.

Die Arzte sollen bei Verwendung von Hormonen und radioaktiven Medikamenten vor-sichtig und zuriickhaltend sein.

10. Krebsvorbeugung ist Schutz vor chronischer Vergiftung durch Stofie und Strahlen.

Prof. Dr. H. R. Schinz 